\title{
Bayesian hierarchichal model for evaluating the risk of vessel strikes on North Atlantic right whales in the SE United States
}

\author{
Christopher J. Fonnesbeck ${ }^{1, *}$, Lance P. Garrison ${ }^{2}$, Leslie I. Ward-Geiger ${ }^{3}$, \\ René D. Baumstark ${ }^{3}$ \\ ${ }^{1}$ Department of Mathematics and Statistics, PO Box 56, University of Otago, Dunedin, New Zealand \\ ${ }^{2}$ National Marine Fisheries Service, Southeast Fisheries Science Center, 75 Virginia Beach Dr., Miami, Florida 33149, USA \\ ${ }^{3}$ Fish \& Wildlife Research Institute, Florida Fish \& Wildlife Conservation Commission, St. Petersburg, Florida 33701, USA
}

\begin{abstract}
A primary factor threatening the recovery of the North Atlantic right whale is the ongoing risk of collision with large ocean-going vessels. Hence, any viable conservation strategy must include mitigation of this risk. In particular, the critical wintering habitat off the Atlantic shores of the southeastern United States overlaps with the shipping routes of some of the region's busiest ports. As a first step in the process of ship strike risk mitigation for this region, we estimated the risk associated with current patterns of shipping traffic, and compared this with estimates of risk for a set of hypothetical alternative routes. As a measure of risk, we selected the co-occurrence of whales and vessels within cells of a $4 \mathrm{~km}$ grid. We performed parametric estimation of whale encounter rate and associated risk within a Bayesian hierarchical model, using data from aerial surveys and the Mandatory Ship Reporting System of the SE United States, along with a selection of environmental covariates. Importantly, we were able to account for annual and monthly variation in encounters in our estimates. All alternative routes provided reduced overall risk, ranging from a 27 to $44 \%$ reduction, relative to the estimated risk of observed traffic. The largest marginal gains in risk reduction were attained by restricting traffic associated with the busiest port, Jacksonville, Florida, but restrictions on all ports achieved the highest reduction. We emphasize the importance of accounting for temporal as well as spatial variation in whale encounter rates, given the migratory behavior of the species.
\end{abstract}

KEY WORDS: Bayesian model - Eubalaena glacialis $\cdot$ Hierarchical model $\cdot$ Right whale $\cdot$ Risk analysis

\section{INTRODUCTION}

The risk of collision with ocean-going vessels is a continuing challenge to the recovery of the endangered North Atlantic right whale Eubalaena glacialis. Numbering perhaps fewer than 400 individuals, important parts of the population's summer and winter habitat coincide with areas of high commercial shipping traffic (Ward-Geiger et al. 2005). Like most longlived mammals, right whale population growth is extremely sensitive to changes in adult survival, par- ticularly for females. It has been suggested that avoiding just a handful of adult female mortality events each year could result in the stabilization of population dynamics (Fujiwara \& Caswell 2001). Hence, preventing collisions with ships - a primary cause of right whale deaths (Kraus 1990, Knowlton \& Kraus 2001) is an important part of the right whale management plan (National Marine Fisheries Service 2005).

Mitigation of ship strike risk is a primary focus of management for North Atlantic right whales on their critical wintering habitat in the southeastern United 
States (SEUS), the only known calving area for the species (Kenney et al. 2001). This can involve altering the movement patterns of large vessel ship traffic through and around right whale critical habitat such that the risk of collision is minimized. One management strategy is to select and enforce shipping traffic routes that result in a relatively low overall risk of encounter with wintering right whales for ships using these routes. This requires some spatio-temporal, possibly dynamic, estimates of whale-vessel encounter probabilities over all candidate shipping routes to major ports in the SEUS, along with some estimate of uncertainty for these probabilities.

It is important to establish the appropriate spatial and temporal scales for the estimation of risk. Both must have sufficient resolution to facilitate the establishment of shipping lanes in space and time, yet be coarse enough to support estimates of adequate precision. Accounting for very small-scale processes (e.g. responses of individual whales to approaching vessels) is problematic, due to the difficulty in collecting the appropriate behavioral data at that scale, and will therefore be avoided. Instead, we will estimate occurrence and quantify risk on a grid at a spatial scale that is suited to the precision of the available data.

This work describes a spatial, hierarchical Bayesian risk model, employing available survey data and predictive covariates to estimate and predict occurrence. This model, in turn, is used to quantify the relative risk associated with candidate routes in a set of alternative shipping lanes. In the absence of estimates of detectability, it is impossible to estimate population size without bias (Pollock \& Kendall 1987, Thompson 2002). Instead, we predict the occurrence of right whale sightings in time and space across the SEUS critical habitat, based on survey data and an associated spatial covariate model for the prediction of whale occurrence. A key advantage of a Bayesian approach to risk analysis is the capacity to model uncertainty in model parameters (and hence, predictions) more realistically, and integrate this uncertainty into our estimates of risk. Where data are sparse, parameters will have wide probability intervals, and these will manifest themselves as reduced certainty in estimates of risk. As new data are collected, the model may be updated in a Bayesian framework to reflect the new information.

\section{STUDY AREA AND DATA}

Our study focused on the risk of right whale collision with ships within the SEUS Mandatory Ship Reporting System (MSRS, Fig. 1). This zone was established in 1999 and ranges from $30^{\circ} 00^{\prime} \mathrm{N}$ to $31^{\circ} 27^{\prime} \mathrm{N}$ latitude, in-

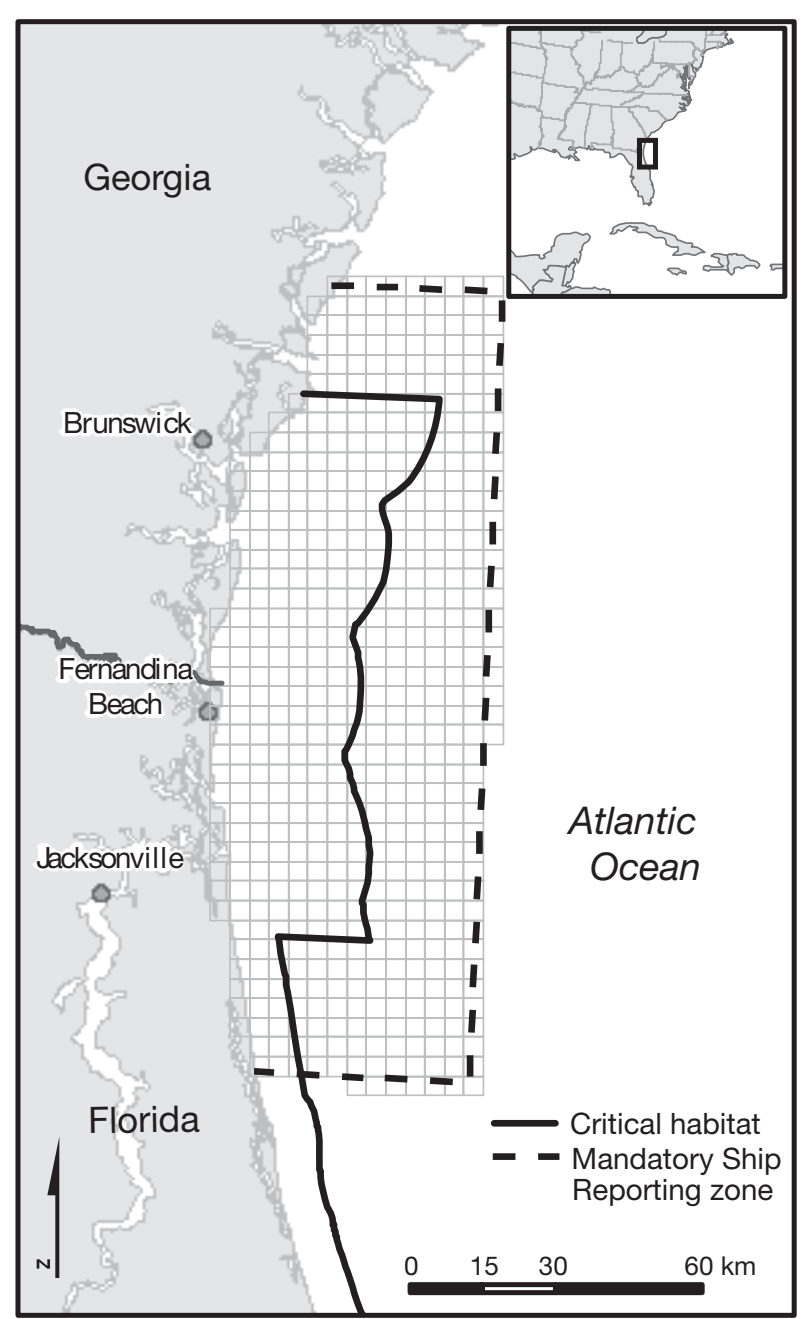

Fig. 1. Southeast United States (SEUS) right whale critical habitat boundary and mandatory ship reporting (MSR) zone, subdivided into $4 \mathrm{~km}$ grid cells

cluding coastal waters within approximately $45 \mathrm{~km}$ of the shore, bounded to the east at $80^{\circ} 51.6^{\prime} \mathrm{W}$ longitude (Silber et al. 2002). All commercial vessels exceeding 300 gross US tons are required to report their position to shore when entering the delineated area (WardGeiger et al. 2005). This southern MSRS zone includes most of the right whale critical habitat in the southeast and is seasonal, operating from November 15 through April 16, coinciding with the calving season.

Though our goal was to estimate risk of collision, we knew relatively little about the small-scale interactions between ships and whales, such as behavioral responses of right whales or sighting probabilities of whales by crew. To date, there is little or no evidence for evasive behaviors of whales in response to approaching vessels, in part because of the difficulties inherent in collecting data at that scale. We thus chose to estimate risk at a relatively coarse scale, identifying 
broader areas of possible co-occurrence that may require management. To this end, we discretized the southeast MSRS zone into a grid composed of $4 \mathrm{~km} \times$ $4 \mathrm{~km}$ cells. We believe this scale is small enough to characterize the heterogeneity in vessel traffic intensity and in habitat variables that may affect right whale distribution, yet large enough to accrue a sufficient number of vessel and whale observations to generate acceptable statistical estimates. The scale is also reasonable considering the precision of the reporting system's requirements for location, which is reported to the nearest whole minute.

Information on the distribution of right whales was derived from aerial surveys, which were conducted throughout the right whale calving season each year. The surveys, flown under similar (good) conditions and using similar aircraft, provided long-term data on right whale distribution within federally designated critical habitat (Fig. 1) and adjacent areas. Keller et al. (2006) describe the distribution and intensity of the first several years (1991 to 1998) of SEUS survey effort. Effort varied through time, but core areas were consistently surveyed. Annually, the greatest effort was expended in critical habitat between Brunswick, Georgia, and south of Jacksonville, Florida, USA. More recently, the survey effort was modified to include the entire MSRS zone.

Survey data consisted of total right whale group sightings $y_{i t}$ and the associated mean aerial survey 'on watch' effort ( $n_{i t}$ in days), summarized by $4 \mathrm{~km}$ grid cell $(i=1, \ldots, N)$ and survey month across all years $(t=$ $1, \ldots, M ;$ December through March). Data were available from seasons 1992 to 1993 through 2004 to 2005; therefore, $t=1$ corresponds to December 1992, while $t=M$ corresponds to March 2005. In addition, 2 key environmental covariates, monthly average sea surface temperature (SST) and bathymetry (mean ocean depth), were used to develop a predictive model for right whale occurrence. To aid in the interpretation of parameter estimates, both bathymetric and temperature data were normalized by subtracting the grand mean and dividing by the standard deviation of the data.

Vessel traffic information was based on MSRS reports during available years (1999-2000 to 2004-2005). Ship routes were estimated based on reported track information provided by the MSRS (Ward-Geiger et al. 2005). In most cases, a straight-line path was assumed between the point-of-entry into the zone and the pilot buoy at the entrance to the port of call. This assumption was made in the absence of additional positional information within the MSRS zone. Routes were summarized over the grid by counting all paths that intersected with each grid cell for each month of the study interval.

\section{MODELING AND ESTIMATION}

It is natural to model counts of surveyed animals as Poisson-distributed random variables. However, preliminary investigation of the right whale survey data revealed severe lack-of-fit under the Poisson assumption. Most of the observations were zeros and ones, with very few counts greater than one. As a result, instead of modeling the expected number of groups, we chose to estimate the probability of encountering one or more groups per month of survey effort. The probability of one or more detections in a given cell $i$ during a given month can be expressed as the complement of the probability of no detections. In turn, the probability of no detections during a given month is the product of the probability of observing no groups $\left(y_{i j}=0\right)$ on each survey day $j$. Here, we assume that these daily probabilities are approximately equal, so this is the daily probability $\operatorname{Pr}\left(y_{i}=0\right)$ raised to the number of survey days $n$ :

$$
\begin{gathered}
\pi_{i}=1-\prod_{j=1}^{n} \operatorname{Pr}\left(y_{i j}=0\right)= \\
1-\left[\operatorname{Pr}\left(y_{i}=0\right)\right]^{n}
\end{gathered}
$$

While we assume that the probability of encounter $\pi_{i}$ is relatively constant within each month, this assumption is likely not valid over longer periods of time and over space, due to seasonal movement by migratory right whales. This leads us to estimate unique encounter probabilities for each cell in each survey month (December to March) in each year (indexed by $t$ ). We characterize spatial variability as a function of physical variables, SST $s$ and bathymetry $d$. Hence, $\pi_{i t}$ is expressed as a hierarchical function:

$\operatorname{logit}\left(\pi_{i t}\right)=\theta+\alpha_{Y} I(\tau \in Y)+\beta_{0} d_{i}+\beta_{1} d_{i}^{2}+\gamma_{0} s_{i t}+\gamma_{1} s_{i t}^{2}$

where $\theta$ is a global mean, $\alpha_{y}$ a year effect, $\beta_{0}$ and $\beta_{1}$ are first- and second-order covariates, respectively, for bathymetry, and $\gamma_{0}$ and $\gamma_{1}$ are first- and second-order covariates, respectively, for SST (second-order effects were included to allow for maxima at intermediate values of the covariates). The indicator function $I(t \in Y)$ returns 1 if survey month $t$ is in year $Y$, and 0 otherwise. To aid the interpretability of year effects, they were modeled as zero-mean normal random variables with precision (inverse variance) $\tau: \alpha_{y} \approx N(0, \tau)$. The logit transformation was applied to constrain estimates of encounter rate to the $(0,1)$ interval.

The likelihood of unit encounter probabilities given observed data was evaluated using a Bernoulli distribution, which describes the distribution of single binary events; hence, $x_{i t}=1$ with probability $\pi_{i t}$, otherwise $x_{i t}=0$. Here, the event in question is any non-zero survey observation: 


$$
x_{i t}=\left\{\begin{array}{lll}
1 & \text { if } & y_{i t}>0 \\
0 & \text { if } & y_{i t}=0
\end{array}\right.
$$

The relationship among model parameters is summarized in Fig. 2.

\section{ESTIMATION OF RISK}

Consistent with the goals of this research, risk was defined as the co-occurrence (in time and space) of whales and vessels. Thus, we sought to identify routes through cells that predict relatively few whale occurrences in each month of the calving season, according to the predicted encounter rates. A reasonable risk function, then, is the vessel track count per cell, weighted by the probability of whale occurrence and summed over all cells and months:

$$
\hat{\rho}=\sum_{t} \sum_{i} \hat{\pi}_{i t} \hat{\mathrm{V}}_{i t}
$$

where $v_{i t}$ is the estimated number of vessels traversing cell $i$ during month $t$, based on MSRS data. Thus, risk is a monotonically increasing function of both vessel density and encounter probability, and is zero for any cells in which either quantity is zero. An obvious and important assumption of this measure is a direct relationship between the encounter rates estimated from survey data and actual encounter rates with ships. These rates are surely not identical, but we assume that they are directly and positively correlated.

To assess the relative risk of alternative ship traffic patterns, we compared 5 alternative shipping routes (Fig. 3), along with the actual MSRS traffic from seasons 1999 to 2000 through 2004 to 2005, using the estimated risk metric above. Each alternative routing describes different restrictions on the paths taken by vessels into each of the 3 major SEUS ports (Brunswick, GA, Fernandina Beach, FL, and Jacksonville, FL): Option 1 restricts Jacksonville traffic to a direct east-west approach from the eastern edge of the MSR,

Fig. 3. Five candidate options for ship routes to the Brunswick (GA), Fernandina (FL) and Jacksonville (FL) ports, showing the relative volumes of traffic (no. of ships) for permissible routes in each (on log scale), along with observed traffic from 1999 to 2000 through 2004 to 2005. Observed: actual shipping traffic; Option 1: Jacksonville traffic restricted to east-west approach, leaving other ports unrestricted; Option 2: adds second southeast-northwest route to Jacksonville, in addition to the east-west approach; Option 3: constrains Jacksonville traffic as in Option 2 and restricts traffic to Fernandina and Brunswick to east-west approaches; Option 4: allows for second southeast-northwest route to both Fernandina and Brunswick, in addition to the restrictions of Option 3; Option 5: restricts traffic to all 3 ports exclusively to their respective east-west approaches

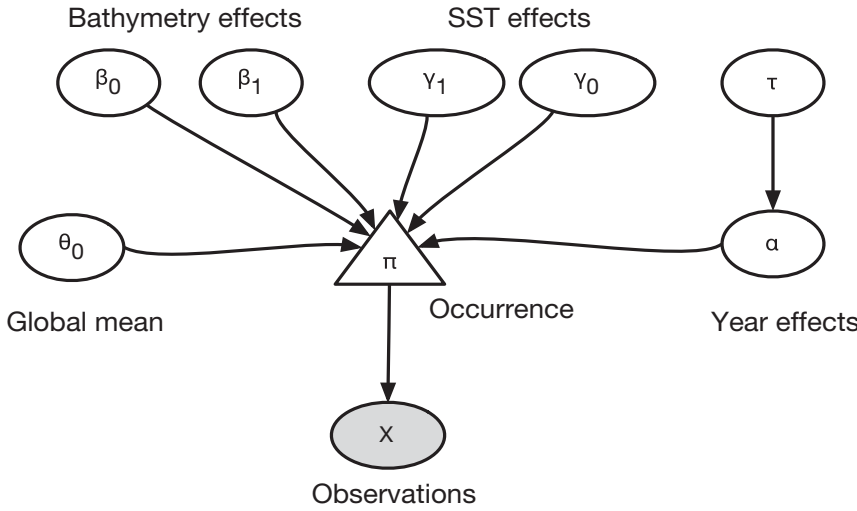

Fig. 2. Hierarchical structure of the predictive model for right whale occurrence. Shaded oval with X represents data, white ovals represent stochastic nodes, and triangle represents calculated nodes. SST: sea surface temperature; $\alpha$ : year effect; $\tau$ : precision (inverse variance) of the year effects; other parameters defined in Table 2

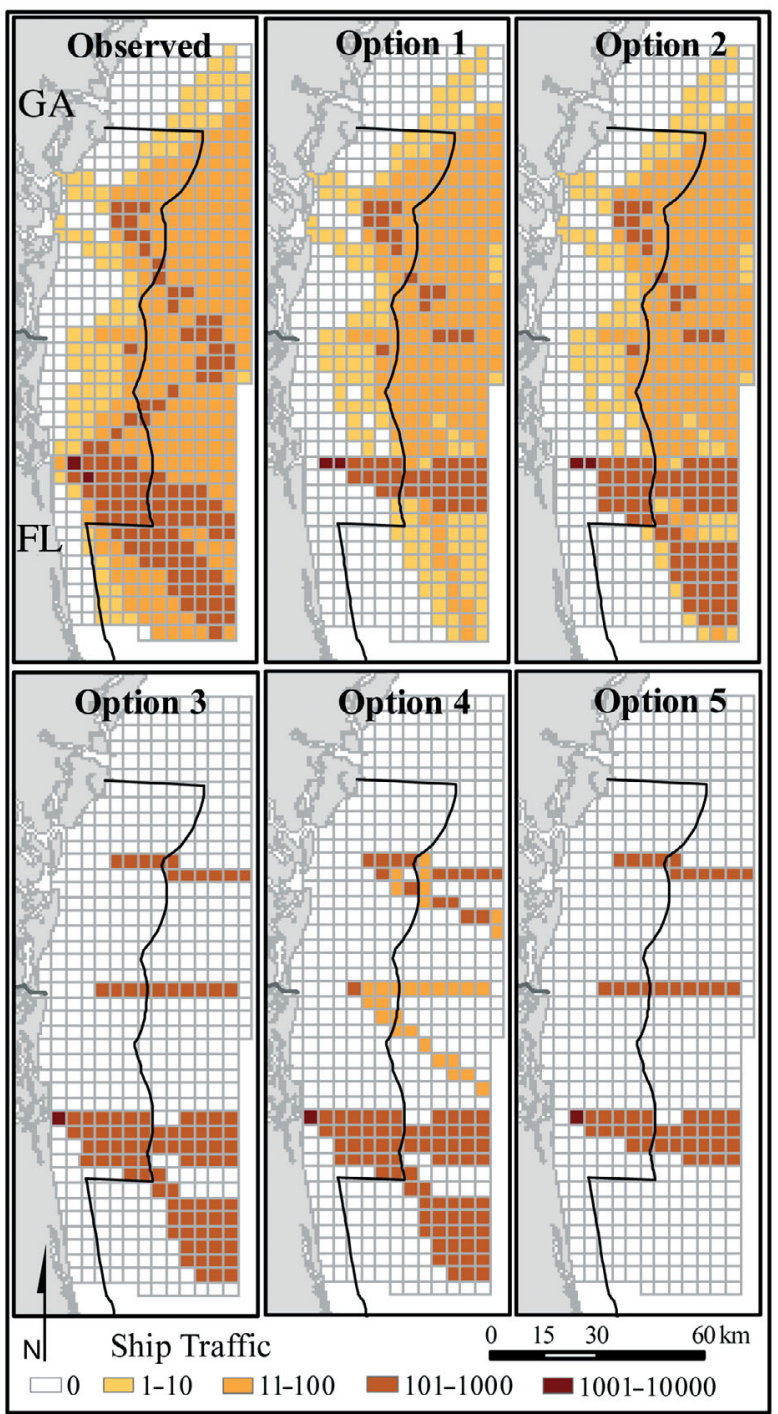


leaving approaches to the other ports unrestricted; Option 2 allows for a second southeast-northwest route to Jacksonville, in addition to the east-west approach; Option 3 constrains Jacksonville traffic as in Option 2, but restricts traffic to Fernandina and Brunswick to east-west approaches from the eastern edge of the MSR; Option 4 allows for a second southeast-northwest route to both Fernandina and Brunswick, in addition to the restrictions of Option 3; and Option 5 restricts traffic to all 3 ports exclusively to their respective east-west approaches. For comparison, the observed MSRS traffic is denoted Option 0. For each alternative, the total volume of shipping was kept constant. Note that for some scenarios, vessel traffic stops several kilometers offshore. This indicates the presence of a Morse Code Alpha buoy, from which ships are typically guided into port by harbor pilots (Ward-Geiger et al. 2005).

Model parameters and expected risk were estimated together in a hierarchical Bayesian model, using a Markov chain Monte Carlo algorithm implemented by the PyMC module (http://pymc.googlecode.com) for the Python programming language (http://python. org). In the absence of strong prior information about model parameters, all parameters were assigned vague prior distributions; logit-linear coefficients were given mean-zero normal priors with low precision (high variance), $\tau=0.001$, while the precision hyperparameter for the year effects was assigned a uniform prior over the interval $(0,100)$. The model was run for 50000 iterations, with 20000 discarded as a burn-in interval, leaving 30000 iterations for inference. Based on inspection of traces, convergence diagnostics and formal goodness-of-fit measures provided by PyMC, there was no evidence of either lack of convergence or lack of fit. Spatial visualization and databases were supported by the GRASS Geographical Information System (GRASS Development Team) and ArcGIS (ESRI).

To assess the importance of the predictor variables described above, we fit 5 different models, each including a different combination of parameters. Along with the full model (all parameters included), we

Table 1. Deviance information criterion (DIC) values and associated model weights corresponding to alternative models comprised of different combinations of predictive covariates. SST: sea surface temperature

\begin{tabular}{|lccc|}
\hline Model & DIC & $\Delta$ DIC & Weight \\
\hline Year + SST + Bathymetry & 7257 & 0 & 1.000 \\
SST + Bathymetry & 7295 & 38 & 0.000 \\
Year + SST & 7459 & 202 & 0.000 \\
Year + Bathymetry & 7620 & 363 & 0.000 \\
Intercept only & 7864 & 607 & 0.000 \\
\hline
\end{tabular}

included an intercept-only model, one that excluded year effects, another that excluded SST effects, and a fifth that excluded bathymetry effects. These models were compared using deviance information criterion (DIC), a Bayesian information-theoretic distance measure (Spiegelhalter et al. 2002). The full model garnered nearly $100 \%$ of the model weight (Table 1 ). Hence, only the results from this model will be presented.

\section{RESULTS}

All alternative routes were estimated to have reduced risk relative to the observed traffic, based on data from 1992 to 1993 through 2004 to 2005 (Fig. 4). There were 3 distinct groups of routes with similar estimates of risk, based on clustering of their posterior marginal distributions. The low risk group included Options 3, 4 and 5, while the moderate risk group included Options 1 and 2. Nominally, Option 4 had the lowest mean cumulative risk. Option 0 (observed traffic) comprised the high risk group. The lowest estimated risk, offered by Option 4, incurred 56.1\% (95\% Bayesian credible interval: 55.9, 56.4) of the risk of the observed traffic. In contrast, the poorest alternative route, Option 1, was estimated to have $73.3 \%$ (72.9, 73.6) the risk of the observed traffic.

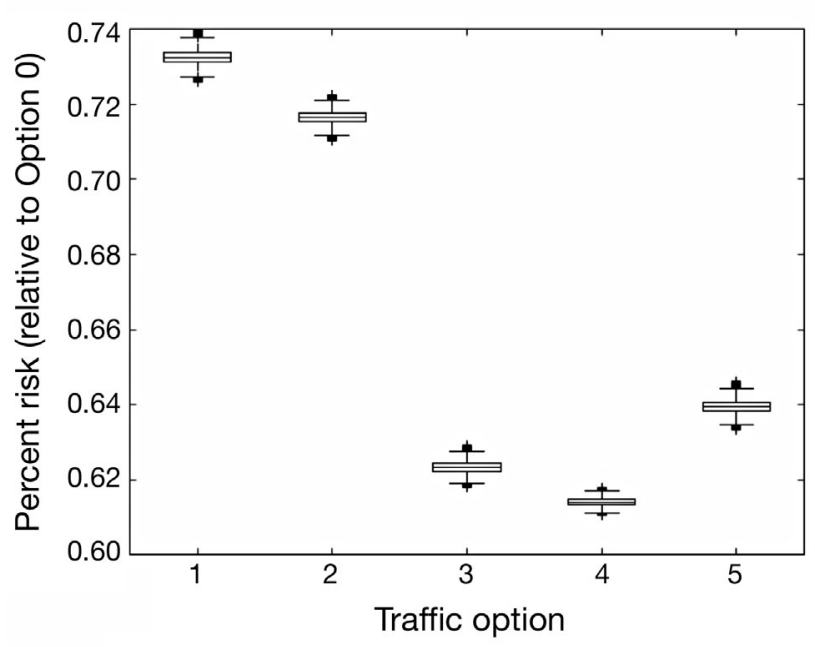

Fig. 4. Boxplots show distributions of the reduction in estimated cumulative risk for 5 alternative patterns of ship traffic relative to observed pattern over a 13-season period (1992-1993 to 2004-2005). Centerline of each plot indicates the median value, boxes the central $50 \%$ density, and whiskers the $96 \%$ Bayesian credible intervals (BCI) for each scenario. Options include: Jacksonville eastern approach only (Option 1); Jacksonville eastern and southeastern approaches only (Option 2); Jacksonville eastern and southeastern approaches, plus Brunswick and Fernandina approaches (Option 3); all approaches (Option 4); and eastern approaches to all ports only (Option 5) 
Table 2. Parameter estimates, variance and 95\% Bayesian credible intervals (BCI) for hierarchical model parameters. All estimates are expressed on the logit scale, except for annual effects variance $\tau^{-1}$ and risk $\rho_{i}$. SST: sea surface temperature

\begin{tabular}{|lcccc|}
\hline Parameter & Mean & SD & BCI 2.5\% & BCI 97.5\% \\
\hline$\theta_{0}$ (global mean) & -5.050 & 0.077 & -5.198 & -4.899 \\
$\tau^{-1}$ (annual effects variance) & 0.048 & 0.028 & 0.010 & 0.099 \\
$\beta_{0}$ (bathymetry, linear) & -0.265 & 0.055 & -0.364 & -0.149 \\
$\beta_{1}$ (bathymetry, quadratic) & -0.398 & 0.050 & -0.503 & -0.309 \\
$\gamma_{0}$ (SST, linear) & -1.263 & 0.077 & -1.409 & -1.110 \\
$\gamma_{1}$ (SST, quadratic) & -0.513 & 0.048 & -0.621 & -0.428 \\
$\rho_{0}$ (risk, Option 0) & 451.8 & 18.7 & 415.8 & 488.8 \\
$\rho_{1}$ (risk, Option 1) & 330.8 & 13.5 & 304.1 & 356.7 \\
$\rho_{2}$ (risk, Option 2) & 323.6 & 13.2 & 297.4 & 349.0 \\
$\rho_{3}$ (risk, Option 3) & 281.5 & 11.3 & 259.5 & 303.7 \\
$\rho_{4}$ (risk, Option 4) & 277.4 & 11.4 & 255.3 & 299.7 \\
$\rho_{5}$ (risk, Option 5) & 288.8 & 11.6 & 266.6 & 311.8 \\
\hline
\end{tabular}

All posterior parameter summary statistics are compiled in Table 2. Covariate model parameter estimates are expressed on the logit scale; for example, the global intercept $\theta_{0}$ mean of -5.050 corresponds to a daily encounter probability of approximately 0.0064 . This is essentially a 'baseline' probability that excludes year effects, and is centered at mean values of both SST and bathymetry. Year effects were often substantial, with $\hat{\tau}=27.23$ corresponding to a variance of 0.048, albeit with large standard errors. Bathymetry and SST model parameters were relatively precise, reflecting the quantity of data informing these estimates.
Linear and quadratic terms for both bathymetry and SST were negative, indicating maxima at intermediate values of both variables.

The estimated probability of encounter varied substantially over time and space. Fig. 5 shows the monthly estimated mean encounter probabilities $\hat{\pi}_{i t}$ for 2 seasons, 1999 to 2000 and 2000 to 2001, as examples of monthly and annual variation.

\section{DISCUSSION}

We employed a Bayesian hierarchical model for the prediction of monthly right whale occurrence as the basis for a risk assessment for large vessel collisions. The hierarchical model afforded an efficient use of spatial covariate information. Specifically, information from each cell at each time period contributed to the estimation of parameters that described the variation in right whale occurrence over time and space. It may be possible to further leverage spatial information by, for example, applying a conditional autoregressive model structure to use observations from adjacent cells to yield more precise estimates of right whale occurrence. In general, we recommend hierarchical modeling for such problems, where parameters can be expressed as

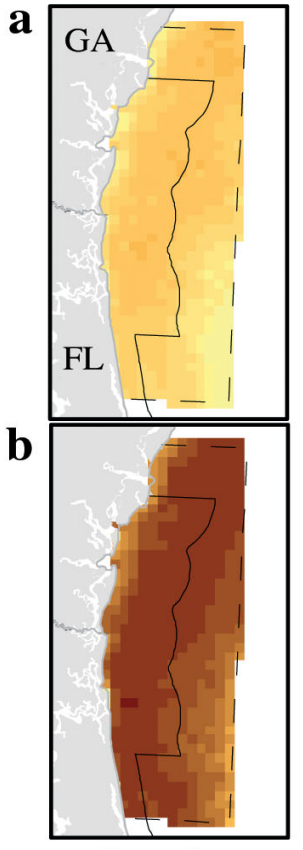

December

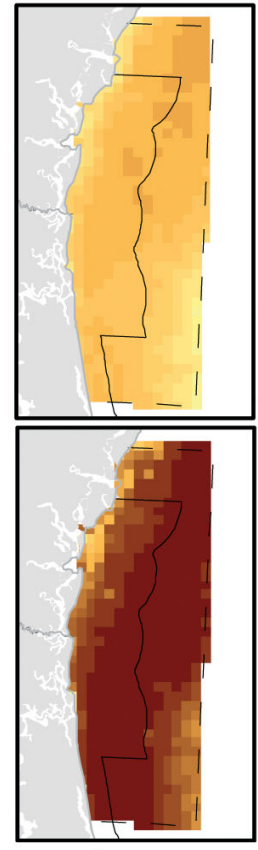

January

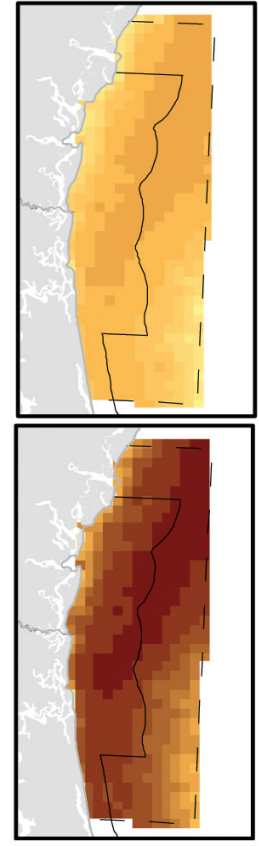

February

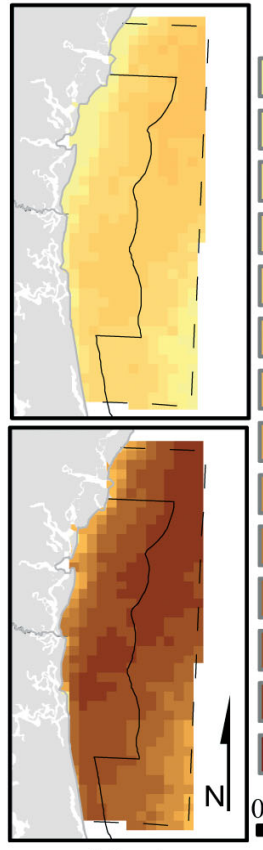

March

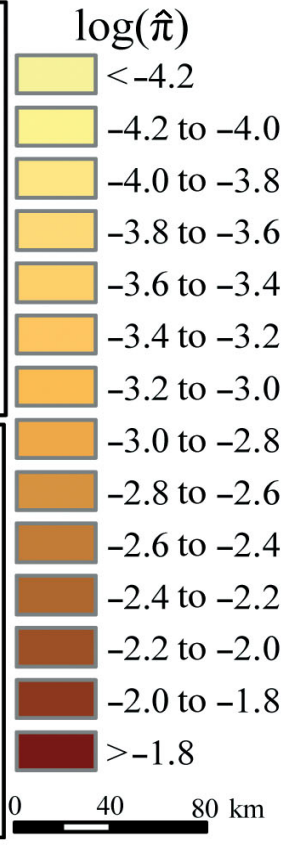

Fig. 5. As an illustration of within- and between-year variation in encounter probability, estimated mean log-probability of encounter $\log (\hat{\pi})$ by month are shown for 2 seasons, (a) 1999 to 2000 and (b) 2000 to 2001 
higher-level functions of other parameters. This avoids either having to ignore heterogeneity and pool data, or separately estimating individual parameters that are obviously related (Gelman et al. 2004).

Right whale occurrence rates showed obvious patterns of temporal variation, both within and among years (Fig. 5, Table 2). Monthly variance is due primarily to migratory movements in and out of the critical habitat throughout the winter (Kenney et al. 2001). Whales are known to move into the southeastern waters in late November and December and begin moving back north in March, following cooler SSTs near the Georgia and Florida coasts within the season (Keller et al. 2006). In addition, there was considerable annual variation in the probability of encounter, particularly with respect to the degree to which whales inhabit the southern extent of the critical habitat during the calving season. Some of this may be attributable to temperature; for example, 2000 to 2001 was a particularly cold year, and these colder conditions coincided with higher encounter rates of whales in the SE part of the critical habitat. This pattern of distribution may have increased vulnerability to ship strike by exposing whales to the dense traffic associated with the large Jacksonville port.

It is perhaps unsurprising that the most restrictive routes were estimated as having the lowest risk, since they constrain the total area of critical habitat exposed to vessel traffic (Fig. 3). Note that the largest reduction in risk comes from imposing travel lanes on the busiest port, Jacksonville (Fig. 4); the distance between the observed traffic risk and the closest alternatives (Options 1 and 2) is entirely attributable to constraining the Jacksonville approach. A smaller, but measurable, reduction comes with subjecting all ports to some defined set of lanes (Options 3, 4, 5). It is interesting that, nominally, the lowest-risk scenario was not the most restrictive (Option 5; third-lowest), but rather Option 4, which allowed 2 alternative lanes for traffic from the east and southeast of each port. This may suggest that the shortest-path (east-west) routes may, on average, traverse cells with relatively high occurrence of right whales. It is important to note, however, that the top 3 scenarios were virtually identical when considering the variation in estimated risk for each, with all system uncertainties taken into consideration.

One of the major limitations in the estimation of risk for current shipping traffic patterns is the quality of shipping data. MSRS data are only estimated paths of incoming vessels which report to the MSRS system. Importantly, it does not include the outbound movement of ship traffic, which could bias the estimates of realized risk. Recently, an improved system of vessel traffic monitoring based on automatic identifications systems (AIS) has been collected. AIS provides a means for ships to report their identification, position, course and speed to other ships and to vessel traffic services (VTS) stations (Harre 2000). This provides very detailed information for all commercial ships exceeding 65 feet $(19.8 \mathrm{~m})$ in length, both outgoing and incoming. As they become available, AIS data could be used to track monthly or annual variation in risk, as well as compliance with implemented lanes. Important future work also includes a comparison with, and possible calibration of, MSRS data.

It is possible that estimates of right whale occurrence presented here are biased by partial availability. Distinct from detection, partial availability occurs when whales are present on a particular survey transect but cannot be seen because they are submerged. In this application, since we are estimating relative (rather than absolute) risk, availability bias is only of concern if we believe such a bias varies over space; this would inhibit valid comparison of encounter rates, and hence risk, among cells in the MSRS zone. One may argue, however, if whales spend significantly more time below surface in particular areas of their habitat, they are less vulnerable to ship strike because of this behavior. Such behavioral differences may exist, for example, depending on whether mothers are accompanied by calves (Szabo \& Duffus 2008).

Most strategies for mitigating ship strike risk for whales involve either vessel speed reductions, or separating whales and vessels spatially by re-routing vessels, or some combination of both (Merrick \& Cole 2007, Vanderlaan et al. 2008). There is some evidence that reduced speed may reduce the fatality rate of collisions (Vanderlaan \& Taggart 2007), suggesting that restrictions on both speed and routes are complementary. However, the full implication of reduced speed on the total risk of collision and mortality is not completely known. Additional research to fully examine the combined effects of speed restrictions and routing measures should be the next step in evaluating the effectiveness of conservation measures. In the absence of additional information, conservation actions based on separating vessels and whales spatially seem most prudent.

This research represents an important early step in estimating ship strike risk for right whales in the SEUS. A Bayesian approach facilitated the adequate characterization of uncertainty in risk that is manifested by uncertainties in model parameter estimates and related, in turn, to the quantity and quality of supporting data. We have shown that the model is flexible enough to be able to evaluate a range of alternative routes. Future model enhancements may include an optimization component, which uses estimated parameters to identify optimal shipping lanes, subject to relevant constraints, such as economic considerations. 
Acknowledgements. We thank 2 anonymous reviewers for the feedback, which greatly improved the quality of this research paper. We gratefully recognize the work and dedication of our colleagues who have spent many long hours conducting right whale aerial surveys in the southeastern US calving area and of the North Atlantic Right Whale Consortium, who curate the aerial survey data. This research was funded by NOAA Fisheries and the Save the Manatee Trust Fund.

\section{LITERATURE CITED}

Besag J, Kooperberg C (1995) On conditional and intrinsic autoregressions. Biometrika 82:733-746

Fujiwara M, Caswell H (2001) Demography of the endangered North Atlantic right whale. Nature 414:537-541

Gamerman, D (1997) Markov chain Monte Carlo: stochastic simulation for Bayesian inference. Chapman \& Hall, London

Gelfand AE, Vounatsou P (2003). Proper multivariate conditional autoregressive models for spatial data analysis. Biostatistics 4(1):11-15

Gelman AE, Carlin JB, Stern HS, Rubin DB (2004) Bayesian data analysis, 2nd edn. Chapman \& Hall/CRC, Boca Raton, FL

Harre I (2000) AIS adding new quality to VTS systems. J Navig 53:527-539

Keller CA, Ward-Geiger LI, Brooks WB, Slay CK, Taylor CR, Zoodsma BJ (2006) North Atlantic right whale distribution in relation to sea-surface temperature in the southeastern United States calving grounds. Mar Mamm Sci 22: 426-445

Kenney R, Mayo C, Winn H (2001) Migration and foraging strategies at varying spatial scales in western North Atlantic right whales: a review of hypotheses. J Cetacean Res Manag (Spec Iss) 2:251-260

Knowlton AR, Kraus SD (2001) Mortality and serious injury of the northern right whales (Eubalaena glacialis) in the

Editorial responsibility: David Hodgson,

University of Exeter, Cornwall Campus, UK western North Atlantic ocean. J Cetacean Res Manag (Spec Iss)2:193-208

Kraus S (1990) Rates and potential causes of mortality in North Atlantic right whales (Eubalaena glacialis). Mar Mamm Sci 6:278-291

Merrick R, Cole T (2007) Evaluation of northern right whale ship strike reduction measures in the great south channel of Massachusetts. NOAA Tech Memo NMFS-NE 202:1-12

National Marine Fisheries Service (2005) Recovery plan for the North Atlantic right whale (Eubalaena glacialis). Technical report, National Marine Fisheries Service, Silver Spring, MD

Pollock KH, Kendall WL (1987). Visibility bias in aerial surveys: a review of estimation procedures. J Wildl Manag 51:502-510

Silber G, Ward L, Clarke R, Schumacher K (2002) Ship traffic patterns in right whale critical habitat: year one of the mandatory ship reporting system. NOAA Tech Mem NMFS-OPR 20

Spiegelhalter DJ, Best NG, Carlin BP, Linde A (2002) Bayesian measures of model complexity and fit. J R Stat Soc Ser C 64:583-639

Szabo A, Duffus D (2008) Mother-offspring association in the humpback whale, Megaptera novaeangliae: following behaviour in an aquatic mammal. Anim Behav 75: 1085-1092

Thompson WL (2002) Towards reliable bird surveys: accounting for individuals present but not detected. Auk 119: $18-25$

Vanderlaan A, Taggart C (2007). Vessel collisions with whales: the probability of lethal injury based on vessel speed. Mar Mamm Sci 23(1):144-156

Vanderlaan A, Taggart C, Serdynska A (2008) Reducing the risk of lethal encounters: vessels and right whales in the Bay of Fundy and on the Scotian Shelf. Endang Species Res 4:283-297

- Ward-Geiger LI, Silber GK, Baumstark RD, Pulfer TL (2005) Characterization of ship traffic in right whale critical habitat. Coast Manag 33:263-278

Submitted: May 23, 2008; Accepted: August 19, 2008

Proofs received from author(s): October 27, 2008 